\title{
Cornual Ectopic Pregnancy: Case Series
}

Baral G, Shakya B, Silwal J

Department of Obstetrics and Gynaecology, Paropakar Maternity and Women's Hospital, Kathmandu.

Received: March 05, 2014; Accepted: February 12, 2015

Three cases of cornual pregnancies encountered within three weeks at Paropakar Maternity and Women's Hospital had diverse presentations. Fortunately, in all three case series, cornual resection was performed successfully. Cornual pregnancy is difficult to diagnose preoperatively with low ultrasonographic sensitivity and is easily confused with tubal ectopic pregnancy or a normal intrauterine pregnancy. Diagnosis before rupture is essential to prevent mortality and potential loss of fertility. The surgical management of diagnosed cornual pregnancy consists of hemostasis, resection, repair and reconstruction.

Keywords: cornual ectopic; cornual pregnancy; cornual resection.

\section{INTRODUCTION}

Cornual pregnancy is a rare subtype of ectopic pregnancy, resulting when implantation occurs in the most proximal part of the fallopian tube. It occurs in approximately 2-4 percent of ectopic pregnancies or one in every 2500 to 5000 pregnancies. ${ }^{1}$ Cornual pregnancies are life-threatening conditions with an associated mortality rate of $2-2.5 \%$ compared to the overall $0.14 \%$ mortality rate for ectopic pregnancy. ${ }^{1,2}$

\section{CASE 1}

Forty years old gravida-2 para- 1 was admitted on $30^{\text {th }}$ May 2014 at 9 pm with suspicion of ectopic pregnancy at 9 weeks and 2 days of amenorrhoea. She had pain abdomen and mild vaginal bleeding seven hours prior to emergency visit. She was hemodynamically stable- pulse $78 \mathrm{bpm}$, blood pressure 120/80 $\mathrm{mmHg}$, hemoglobin $11.9 \mathrm{~g} / \mathrm{dl}$, soft abdomen and brownish vaginal discharge. Urine for pregnancy test was positive with serum $\beta$-hCG 34,401 $\mathrm{mIU} / \mathrm{ml}$ and there were subserous fibroid with heterogenous lesion at right adnexa sized $3.2 \times 2.5 \mathrm{~cm}$ in ultrasonography. She was kept under conservative management. Repeat serum $\beta$-hCG after 48 hours was $31,000 \mathrm{~m}$ IU/ml and ultrasonography revealed gestational sac of seven

\section{CORRESPONDENCE}

Dr Gehanath Baral

Department of Obstetrics and Gynaecology,

Paropakar Maternity and Women's Hospital, Kathmandu. Email: gehanath@gmail.com

Phone: +977-9841228829 weeks with fetal pole and cardiac activity at cornual junction suggesting cornual pregnancy. Emergency laparotomy was done with right cornual resection and repair with left tubal ligation under subarachnoid block. There was hemoperitoneum of about $50 \mathrm{ml}$, left cornual pregnancy sized $6 \times 4 \mathrm{~cm}$ with trickling blood, and round ligament was in the middle at its base. There were multiple subserous fibroids $3 \times 3 \mathrm{~cm}$ near cornual ectopic area and another one $4 \times 4 \mathrm{~cm}$ at anterior wall of uterus. Uterus was soft and enlarged. Left tube and both ovaries were normal. Total blood loss was $200 \mathrm{ml}$. Her post-operative period was uneventful and discharged on $7^{\text {th }}$ postoperative day. Histopathology was reported as cornual ectopic pregnancy.

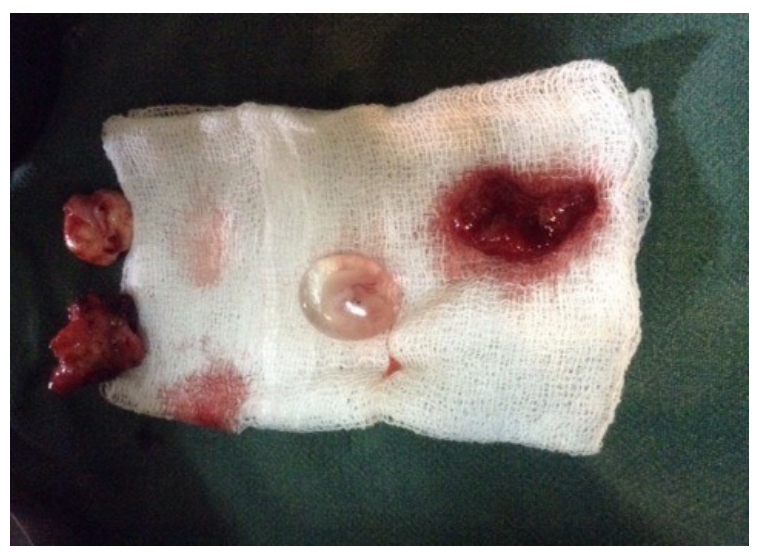

Figure 1. Case 1: Intact gestational sac of cornual ectopic pregnancy. 


\section{CASE 2}

Thirty three years old gravida- 2 para- 1 at seven weeks of amenorrhoea was admitted on $7^{\text {th }}$ June, 2014 at 8.15 PM who had vaginal bleeding following failed surgical termination one week ago at health post and pain abdomen 17 hours prior to emergency visit which was severe and sudden in onset. This was a referred case from Bir Hospital as ultrasonogaphy revealed mixed echogenic lesion of $8.6 \times 5.7 \mathrm{~cm}$ with minimal vascularity at left adnexa and anterior to uterus, and moderate amount of collection in peritoneal cavity. Patient was pale, pulse $110 \mathrm{bpm}$, blood pressure 140/70 mmHg. Abdomen was tender with rebound tenderness without vaginal beeding on speculum examination. Urine for pregnancy test was positive. Patient underwent emergency laparotomy with left cornual resection under subarachnoid block. There was hemoperitoneum of about $2000 \mathrm{ml}(600$ $\mathrm{ml}$ blood $+1400 \mathrm{ml}$ clots). There was ruptured left cornual pregnancy just lateral to the round ligament. Uterus was bulky, right tube and bilateral ovaries were normal. Patient received 2 units blood transfusion intra-operatively and 2 units post-operatively. Her post-operative period was uneventful except for low-grade fever on first and second postoperative day and was discharged on seventh post-operative day. Histopathology report revealed cornual ectopic pregnancy.
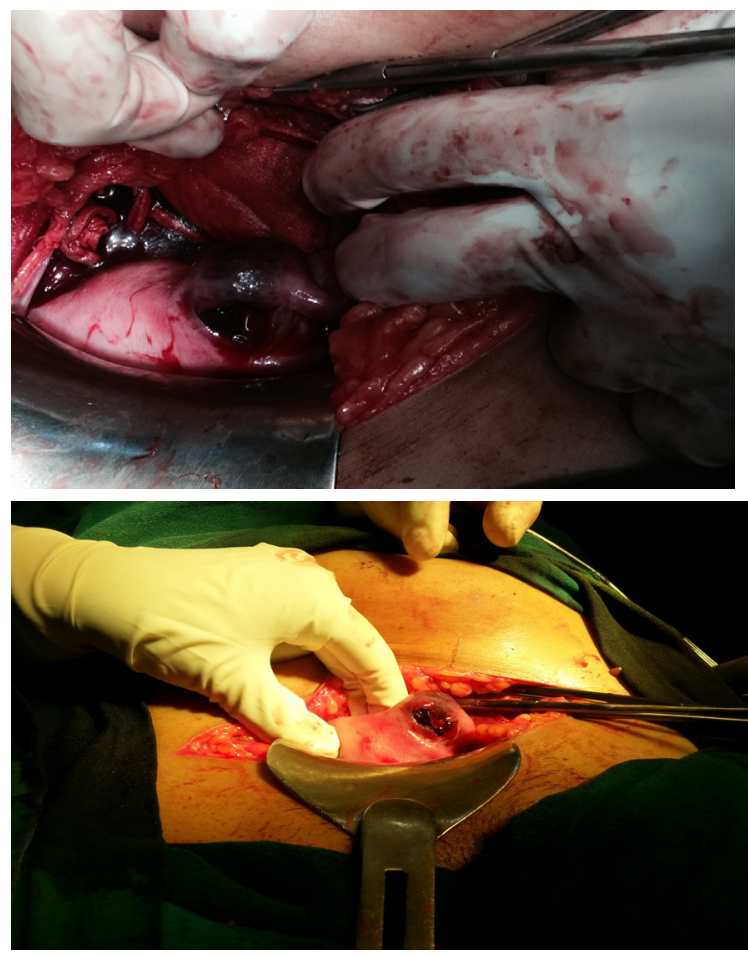

Figure 2-3. Case 2: Cornual ectopic pregnancy.

\section{CASE 3}

Twenty years old primigravida of around 16 weeks of amenorrhoea was admitted through emergency department on $18^{\text {th }}$ June, 2014 at $8 \mathrm{pm}$ as she had history of fall from stairs and vaginal bleeding three hours prior to admission. Her pulse was $78 \mathrm{bpm}$, blood pressure 90/60mmHg. Abdomen was soft, mild tender and uterus was 14-16 weeks size. There was no vaginal bleeding, cervical os was closed and no forniceal tenderness. Patient was managed conservatively. Ultrasonography revealed 17 weeks intrauterine fetal death, fetal ascites and anteriorly situated placenta. The management plan decided as medical termination of pregnancy with misoprostol $200 \mathrm{mcg}$ vaginally 4 doses 6 hours apart. Since she did not respond, she again received $400 \mathrm{mcg}$ misoprostol 4 doses 6 hours apart. Next day, manual vacuum aspiration was tried in operation theatre and no tissue was obtained. Pelvic examination under anesthesia revealed separate mobile mass next to the uterus of equal size. Then with the suspicion of abdominal pregnancy, emergency laparotomy was done under general anesthesia. Per operative finding revealed right cornual pregnancy with engorged vessels over its surface lateral to round ligament and a short pedicle, $50 \mathrm{ml}$ of hemoperitoneum, enlarged uterus with perforation $(3 \times 2 \mathrm{~cm})$ at anterior uterine wall of corpus (iatrogenic) and normal tubes and ovaries. Right cornual resection and repair along with repair of uterine perforation performed. Cut section revealed dead macerated male fetus and intact sac with about $50 \mathrm{ml}$ of amniotic fluid. Operative blood loss was $200 \mathrm{ml}$. Her post-operative period was uneventful and was discharged on seventh postoperative day. Histopathology report revealed cornual ectopic pregnancy.

In all of the case series, the known risk factors like pelvic inflammatory disease, previous ectopic or tubal/pelvic surgery, use of IUCD, artificial reproductive technique or smoking habit were absent. 

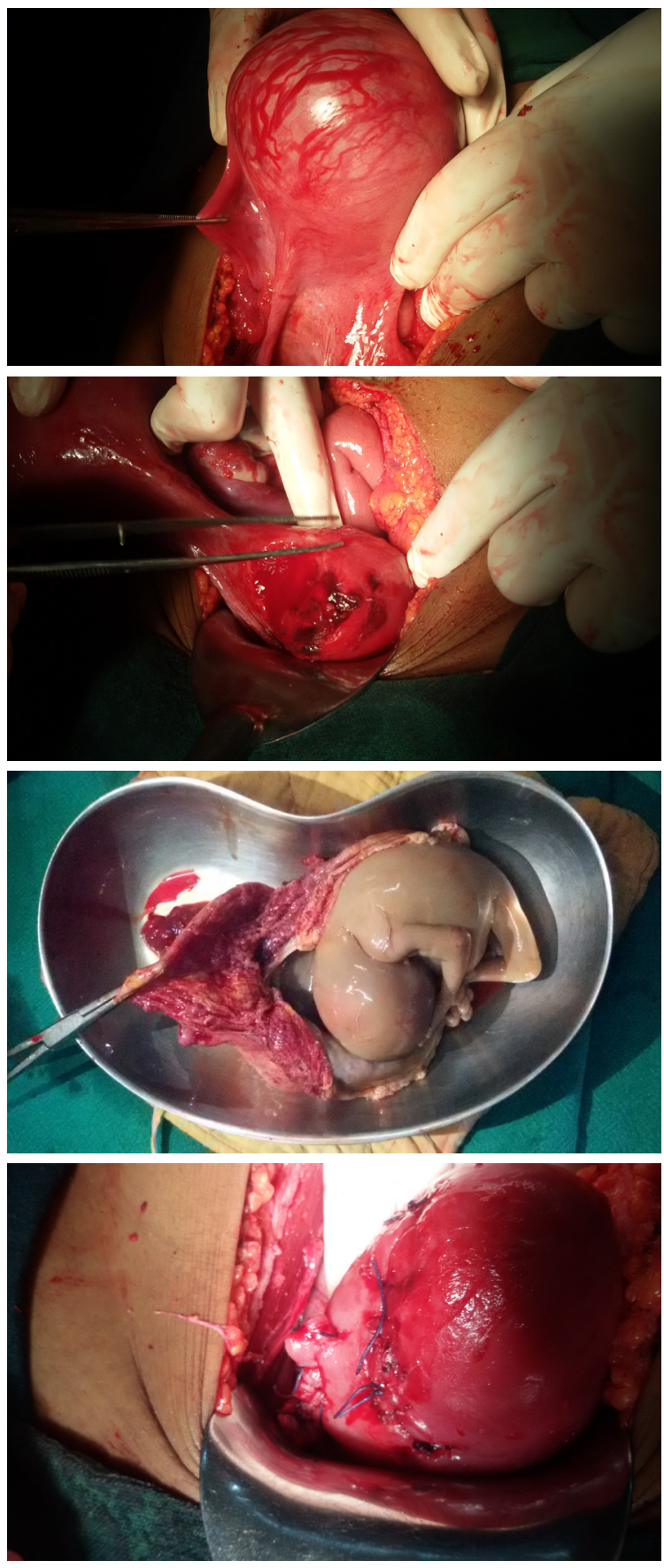

Figure 4-7. Case 3: 16 weeks cornual ectopic pregnancy.

\section{COMMENT}

Each of the case series of cornual pregnancy had diverse presentations. Women over 40 years of age have a $3-4$ fold increase in the risk for developing an ectopic pregnancy compared to women aged 15-24 years. ${ }^{3}$ In our study, case 1 was 40 years the other two were 33 and 20 years.

The ultrasound picture of cornual ectopic can be very similar to that of an early pregnancy in a bicornuate uterus or a fibroid uterus. ${ }^{3}$ In case 1 , it was associated with fibroid uterus missing the cornual pregnancy. Even with a high index of suspicion and advances in sonography, including transvaginal sonography and serum $\beta$-hCG, cornual pregnancy remains the most difficult type of ectopic pregnancy to diagnose due to the low sensitivity and specificity of symptoms and imaging.

Cornual pregnancies show fewer early symptoms due to the stretching potential of the uterine wall. ${ }^{4}$ The site of implantation also makes the pregnancy difficult to differentiate from an intrauterine pregnancy on ultrasound. ${ }^{3,5}$ Moreover, the assessment of the uterine size is rarely helpful and cervical excitation is not a specific sign in cornual ectopic. ${ }^{3}$ As was seen in our third case, 16 weeks cornual pregnancy was missed by clinical and ultrasonography examination as the pregnancy may appear to be in an intrauterine location. Further the clinical examination was limited by abdominal wall tenderness due to fall injury.

Cornual ectopics are associated with high risk of rupture that could occur as late as $10-16$ weeks gestation. ${ }^{3}$ Rupture of a cornual ectopic at that late gestation can cause profuse intraperitoneal bleeding which can be life threatening. As contrast to case 2 in which rupture occurred at seven weeks gestation requiring four units of blood transfusion, patient had presented late to our hospital and moreover, patient was even tried for surgical termination of pregnancy. Fortunately in case 3, there was no rupture of 16 weeks cornual pregnancy despite being tried for misoprostol induction.

Overall, cornual pregnancy is one of the most dangerous type of ectopic pregnancies with high maternal mortality and cannot be solely relied on findings of abdominal ultrasound. Diagnosis is done with transvaginal ultrasound: 1) an empty uterine cavity, 2) a chorionic sac seen separately and $>1 \mathrm{~cm}$ from most lateral edge of uterine cavity, and 3) a thick myometrial layer surrounding the chorionic sac. Laparoscopy may be needed to confirm diagnosis but in case of massive intraperitoneal bleeding immediate laparotomy must be done. The surgical principle for the management of diagnosed cornual pregnancy can be described by mnemonic HR3, 
which stands for Hemostasis, Resection, Repair and Reconstruction. The management of cornual ectopic pregnancy depends upon extent of trauma and desire of patient to preserve fertility. It can be done with systemic methotrexate or conservative laparoscopic technique in selected cases. Cornual resection and repair of the defect by laparotomy remains the standard conservative surgical procedure for many surgeons. In some cases, where uterine rupture had occurred or a very large cornual pregnancy is present hysterectomy may be required. A cornual resection and salpingectomy is done by first ligating the ascending uterine vessels where they approach the cornua. The pregnancy site is excised in a V-shaped manner, and the myometrium is approximated with figure of eight closure using no. 0 delayed absorbable suture. The round ligament is cut and resutured to the cornu and the uterine serosa by use of interrupted sutures. The round and broad ligaments are brought over the incision with mattress suture (the modified Coffey Suspension), and an additional interrupted sutures of no. 2-0 or no. 3-0 delayed absorbable sutures can be used to secure the serosa of the round ligament to the serosa of uterus to maintain the operative site in a permanent retroperitoneal position.

Traditionally, cornual ectopic pregnancies were treated with hysterectomy. The current gold standard for a cornual pregnancy is a diagnostic laparotomy followed by a cornual wedge resection. Recent surgical advances have demonxstrated that operative laparoscopy is a possible alternative in a stable patient with no suspicion of rupture. ${ }^{6}$ Fortunately, in all three case series, cornual resection was performed successfully.

Repeat sonographic assessment is essential even in light of a "normal" intrauterine pregnancy if a patient's symptoms are consistent with ectopic pregnancy. Diagnosis before rupture is essential to prevent mortality and potential loss of fertility. ${ }^{7}$

\section{CONCLUSIONS}

Clinical differentiation of cornual pregnancy from tubal ectopic pregnancy or a normal intrauterine pregnancy is often difficult due to low ultrasonographic sensitivity. Since, symptoms are often nonspecific and diagnostic imaging inconclusive, correct diagnosis of an advanced extrauterine pregnancy warrants a high index of suspicion.

\section{DISCLOSURE}

The authors report no conflicts of interest in this work.

No violation of human rights and safety.

Funding: Nil

\section{REFERENCES}

1. Radwan F, Steel M. Review Management of cornual (interstitial) pregnancy. Obstet Gynaecol. 2007;9(4):249-55.

2. Wright CT. Sonographic evaluation of interstitial (cornual) ectopic pregnancy. J Diagn Med Sonogr. 2008;24:374-9.

3. Shendy M, Atalla R. Modern management of cornual ectopic pregnancy, ectopic pregnancy - modern diagnosis an management, Dr. Michael Kamrava (Ed.), ISBN: 978-953 307-648-5. Available from: http://www.intechopen.com/ books/ectopic-pregnancy

4. Molinaro TA, Barnhart KT. Ectopic pregnancies in unusual locations. Semin Reprod Med. 2007;25(2):123-30.
5. Wright CT. Sonographic evaluation of interstitial (cornual) ectopic pregnancy. J Diagn Med Sonogr. 2008;24:374-9.

6. Auslender R, Arodi J, Pascal B, Abramovici H. Interstitial pregnancy: early diagnosis by ultrasonography. Am J Obstet Gynecol. 1983;146(6):717-8.

7. Walid MS, Heaton RL. Diagnosis and laparoscopic treatment of cornual ectopic pregnancy. Ger Med Sci. 2010;8:1-4.

8. Myran D. Double miss by ultrasound of a cornual ectopic pregnancy. UWOMJ. 2013;82(S1):12-13. 